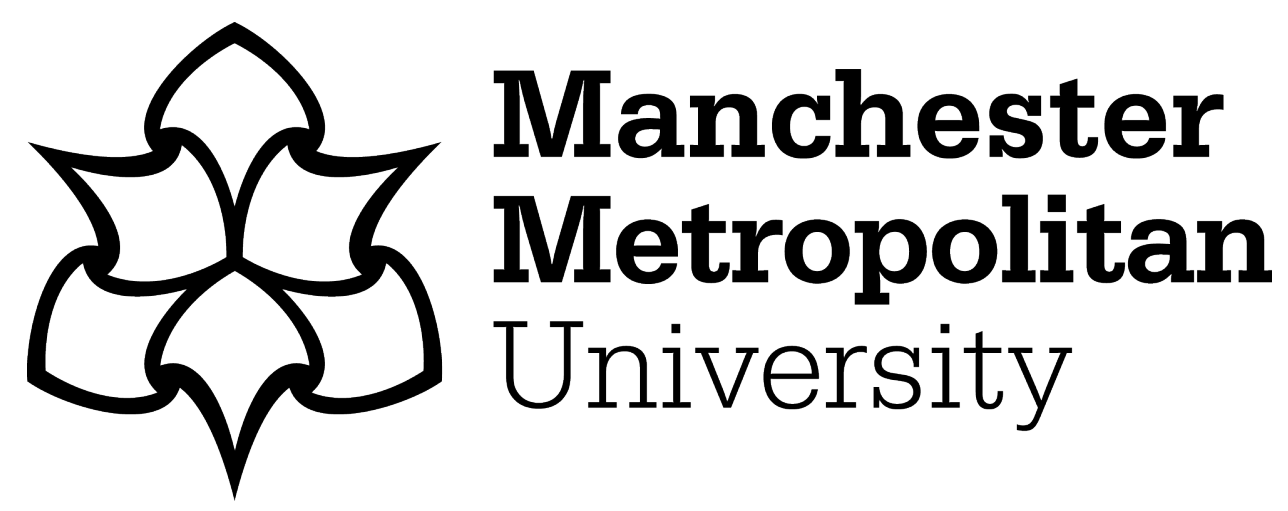

Greenhalgh, L, Rashid, M, Linton, PE ORCID logoORCID: https://orcid.org/0000-0002-1106-4988 and Shaw, KJ ORCID logoORCID: https://orcid.org/0000-0001-9241-4195 (2020) A Microfluidic Device for Nucleic Acid-Based Analysis of Helicobacter pylori and Antibiotic Susceptibility Testing. In: 7th International Conference on the Development of Biomedical Engineering in Vietna, (BME7), 27 June 2018 - 29 June 2018, Ho Chi Min City.

Downloaded from: https://e-space.mmu.ac.uk/624085/

Version: Accepted Version

Publisher: Springer

DOI: https://doi.org/10.1007/978-981-13-5859-3_65

Please cite the published version 


\title{
A Microfluidic Device for Nucleic Acid-Based Analysis of Helicobacter pylori and Antibiotic Susceptibility Testing
}

\author{
Leah Greenhalgh, Mamun Rashid®@, Patricia E. Linton®®, \\ and Kirsty J. Shaw
}

\begin{abstract}
Rapid diagnosis of bacterial infections enables earlier implementation of appropriate and effective treatment regimens, therefore improving patient outcomes. We have previously demonstrated a microfluidic device for nucleic acid-based analysis of Helicobacter pylori, which can cause peptic ulcers and increase the risk of stomach cancer. One of the major challenges to overcome has been the use of challenging real-world samples, e.g. stool, and urine allowing use at the point-of-care. Here we present results for bacterial identification as well as initial studies on the use of screen-printed electrodes (SPE) for miniaturised electrochemical detection for determining antibiotic susceptibility of pathogenic Escherichia coli. Resazurin was used as an indicator of bacterial viability, with reduction of resazurin demonstrating continued cell growth. Differential pulse voltammetry was used to measure potential reduction and showed measurement at $-0.58 \mathrm{~V}$ or $-0.38 \mathrm{~V}$ to be the most discriminatory. A fixed voltage of $-0.58 \mathrm{~V}$ was then used to monitor current changes as a function of increasing gentamycin (antibiotic) concentration, with the results showing a significant reduction in current with increasing amounts of gentamycin (ANOVA, $p<0.001$ ), within $90 \mathrm{~min}$. The ability to integrate diagnosis with antibiotic susceptibility testing would allow administration of timely and specific treatment to patients with serious infections in low-resource settings.
\end{abstract}

\section{Keywords}

Microfluidics - Bacterial infection - Antibiotic susceptibility

\section{Introduction}

Bacterial infections affect hundreds of thousands of people annually worldwide. Rapid diagnosis of bacterial infections enables earlier implementation of appropriate and effective treatment regimens, therefore improving patient outcomes. Patient samples are preferentially collected using non-invasive methods, i.e. urine or stool samples, for both ease of collection and improved patient compliance. However, both sample types present challenges for analysis in terms of low target analyte concentrations and heterogeneity. Bacterial strain identification is important as different strains can have varying pathogenicity, for example, the phenotype of Helicobacter pylori (H. pylori) that expresses cytotoxin-associated protein (CagA) causes a higher degree of acute inflammation and is three times more likely to lead to gastric carcinogenesis than $H$. pylori strains which do not express CagA [1]. We have previously demonstrated a microfluidic device for nucleic acid-based analysis of $H$. pylori, from clinical stool samples, which identified the bacterial infection and whether or not it expressed the pathogenic CagA [2].

The growing emergence of antibiotic resistance is a global concern and has been identified as a high priority for the World Health Organisation [3]. Therefore the ability to not only identify the type of infection but also which antibiotics can be prescribed to successfully treat the infection (i.e. antibiotic susceptibility) is crucial to improve clinical outcomes. The use of microfluidics to integrate these steps together offers the potential to create cost-effective diagnostics for point-of-care applications.

Resazurin has been commonly used as a redox indicator to measure the metabolic rate of bacteria, which can be indicative of antibiotic resistance/susceptibility. In aqueous buffer solution, resazurin (blue) undergoes an irreversible two-electron reduction to resorufin (pink) due to the elimination of oxygen from the dye molecule. An additional two electron reversible reduction process to convert resorufin to
L. Greenhalgh · M. Rashid · P. E. Linton - K. J. Shaw $(\bowtie)$ Manchester Metropolitan University, Manchester, M1 5GD, UK e-mail: k.shaw@mmu.ac.uk 


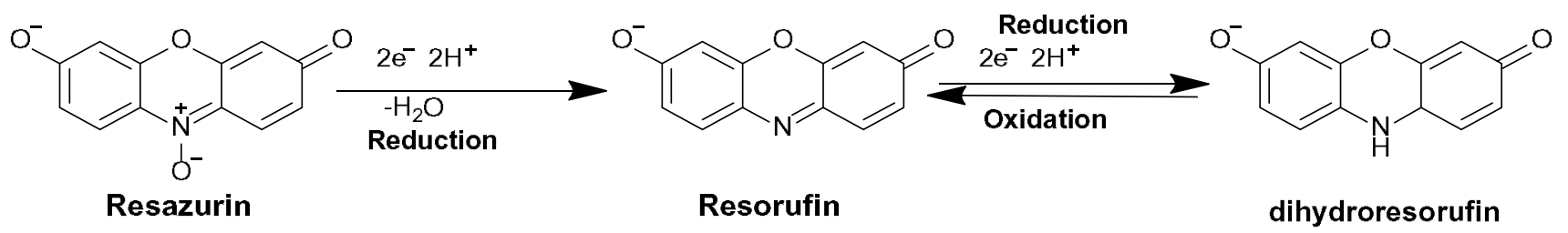

Fig. 1 Reduction mechanism of Resazurin in aqueous buffer medium [4]

dihydroresorufin (colourless) can also occur (Fig. 1). Traditionally reduction of resazurin has been monitored using colourimetric or fluorescence assays which vary in sensitivity.

Here we present work on bacterial identification and initial studies on the use of screen-printed electrodes (SPE) for miniaturised electrochemical detection for determining antibiotic susceptibility of pathogenic bacteria.

\section{Experimental}

Solidworks ${ }^{\circledR}$ was used to produce 3D printed moulds. Polydimethylsiloxane (PDMS) (Sylgard 182, Dow Corning) was then cast into the moulds to create the microfluidic devices (Fig. 2).
Electrochemical measurements were performed using a potentiostat (EmStat-PalmSens, NL) and a three-electrode setup. For off-chip electrochemical experiments, $\mathrm{CH}$ gold electrodes (diameter $2 \mathrm{~mm}$ ) were used with Pt counter and $\mathrm{Ag} / \mathrm{AgCl}$ reference electrodes. For on-chip electrochemical experiments, screen printed carbon working electrodes (diameter $1.5 \mathrm{~mm}$ ) were used with a screen printed Pt counter and $\mathrm{Ag} / \mathrm{AgCl}$ reference electrode (Fig. 3). Both working electrodes were scanned using differential pulse voltammetry (DPV) Resazurin (Sigma-Aldrich, UK) was used as the redox indicator.

Bacterial cultures (Escherichia coli and Staphylococcus aureus) used for control experiments were grown up in nutrient broth (Fisher Scientific, UK) overnight at $37^{\circ} \mathrm{C}$. Gentamycin (Merck, UK) was used as an example antibiotic. Clinical stool samples were obtained from NHS Chesterfield Laboratories; samples were fully anonymized and were
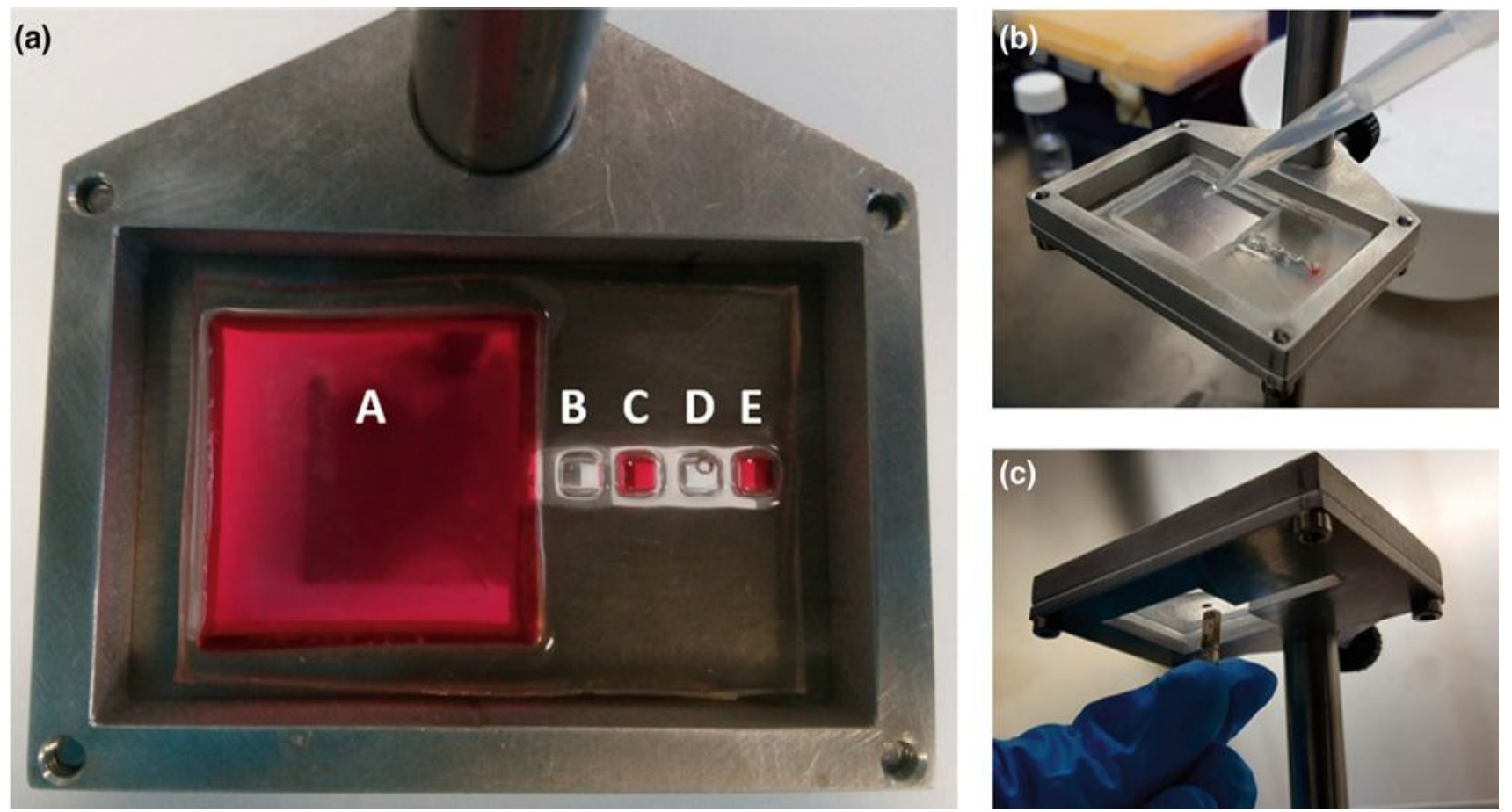

Fig. 2 a Overview of the design of the microfluidic device for bacterial identification loaded with food dyes to clearly indicate (A) sample chamber (pre-loaded with superparamagnetic particles (PMPs) and powdered guanidine hydrochloride (GuHCl), (B \& D) mineral oil, (C) GuHCl wash and (E) elution/amplification chambers; b sample addition and c movement of PMPs using an external $\mathrm{NdFeB}$ magnet from chamber $A$ to $\mathrm{E}$ 


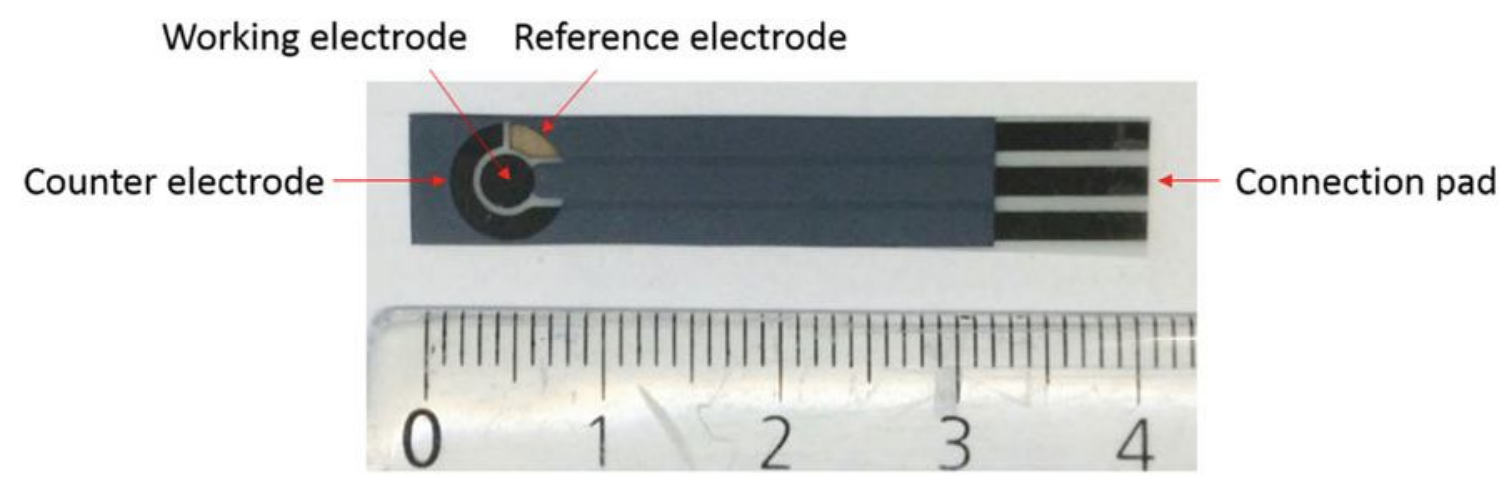

Fig. 3 Screen-printed electrode

selected on the basis that they had previously been tested for H. pylori during routine clinical testing.

\section{Results and Discussion}

Chemical lysis of bacterial cells was achieved on-chip using guanidine hydrochloride powder stored in the sample chamber, and reconstituted upon sample addition. Lysis efficiencies of 80.2 and $69.3 \%$, respectively, for $E$. coli (Gram negative) and $S$. aureus (Gram positive) were demonstrated. DNA extraction efficiencies, using $E$. coli as a model organism, were shown to be greater $(\sim 75 \%)$ when smaller amounts of DNA were present, which is ideal for dealing with low levels of infection in clinical specimens. The use of multiple wash chambers (Fig. 2, chambers B-D) was shown to be necessary when analysing stool samples to provide DNA of sufficient purity for amplification of target markers: Ure $\mathrm{C}$ (for identification of $H$. pylori) and CagA (indicator of severe pathogenic phenotype) [2].

As resazurin and resorufin have different electrochemical signals in aqueous buffer solution, by using differential pulse voltammetry it is possible to identify the oxidation/reduction states of resazurin and thus specify whether the target bacteria is susceptible or not [5]. Figure 4, shows the differential pulse voltammagram recorded during the reduction of resazurin in the three electrode configuration. It is observed that the two electron reduction of resazurin to resorufin occurs at $-0.38 \mathrm{~V}$ (Peak I). An additional two electron reversible reduction process is observed at $-0.58 \mathrm{~V}$ (Peak II) that corresponds to formation of dihydroresorufin in the same aqueous medium.

A fixed voltage of $-0.58 \mathrm{~V}$ was then used to monitor current changes as a function of increasing gentamycin concentration $(10,20,30,40$ and $50 \mathrm{mg} / \mathrm{mL})$, with the results showing a significant reduction in current with increasing amounts of gentamycin (ANOVA, $p<0.001$ ), within 90 min (Fig. 5). In Fig. 5, it was observed that, both

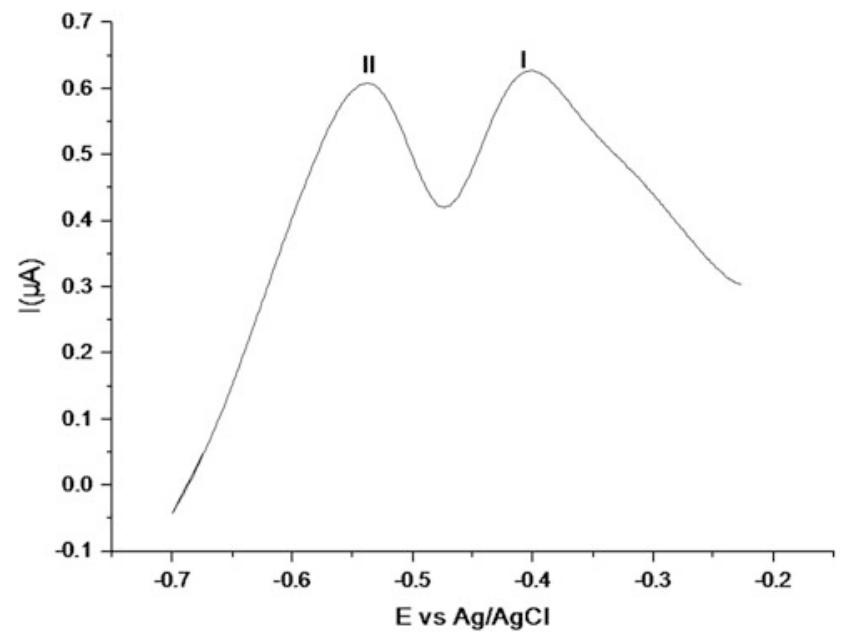

Fig. 4 Representative differential pulse voltammagram recorded during the reduction of buffered resazurin solution $(1 \mathrm{mM})$

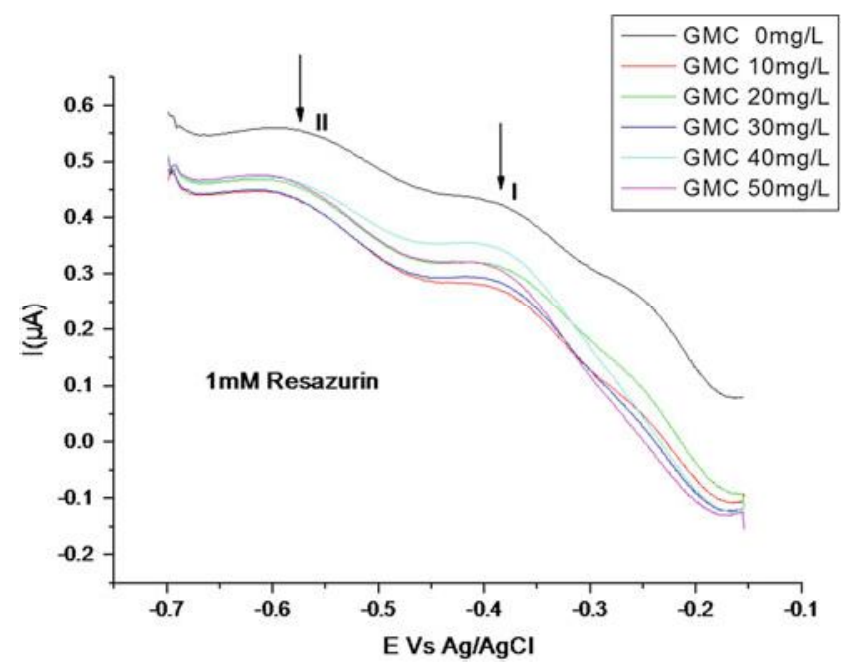

Fig. 5 Representative differential pulse voltammagrams obtained from cultured $E$. coli for 80 min with resazurin and gentamycin (GMC) 
peaks I and II decreased systematically as metabolism was affected by the antibiotic, indicating antibiotic susceptibility.

\section{Conclusions}

The ability to integrate diagnosis with rapid antibiotic susceptibility testing would allow administration of timely and specific treatment to patients with serious infections in low-resource settings. The work presented here shows how PDMS microfluidic devices can be used for identification of bacterial infections (including phenotypic indications) using genetic testing and also monitoring of the metabolic state of the bacteria using SPEs within $90 \mathrm{~min}$.

Conflict of Interest The authors declare no conflict of interest.

\section{References}

1. Parsonnet, J., Friedman, G.D., Orentreich, N., Vogelman, H.: Risk of gastric cancer in people with CagA positive or CagA negative $H$. pylori infection. Gut 40, 297-301 (1997)

2. Mosley, O., Melling, L.M., Tarn, M.D., Kemp, C., Esfahani, M.M.N., Pamme, N., Shaw, K.J.: Sample introduction interface for on-chip nucleic acid-based analysis of Helicobacter pylori from stool samples. Lab Chip 16(11), 2108 (2016)

3. World Health Organisation. http://www.who.int/en/news-room/factsheets/detail/antibiotic-resistance. Accessed 10th May 2018

4. Khazalpour, S., Nematollahi, D.: Electrochemical study of Alamar Blue (resazurin) in aqueous solutions and room-temperature ionic liquid 1-butyl-3-methylimidazolium tetrafluoroborate at a glassy carbon electrode. RSC Adv. 4, 8431-8438 (2014)

5. Besant, J.D., Sargent, E.H., Kelley, S.O.: Rapid electrochemical phenotypic profiling of antibiotic-resistant bacteria. Lab Chip 15, 2799-2807 (2015) 